\title{
Synthesis models in the VO framework
}

\author{
M. Cerviño ${ }^{1,4}$, E. Terlevich ${ }^{2}$, R. Terlevich ${ }^{2}$, C. Rodrigo-Blanco ${ }^{3,4}$, \\ V. Luridiana ${ }^{1,4}$, A. López ${ }^{2}$ and E. Solano ${ }^{3,4}$ \\ ${ }^{1}$ Instituto de Astrofísica de Andalucía (IAA-CSIC), Camino bajo de Huétor 50, 18008 Spain \\ email: mcs@iaa.es, vale@iaa.es \\ ${ }^{2}$ Instituto Nacional de Astrofísica Óptica y Electrónica, Puebla, México \\ email: eterlevi@inaoep.mx, rjt@inaoep.mx, allopez@inaoep.mx \\ ${ }^{3}$ Laboratorio de Astrofísica Espacial y Física Fundamental (LAEFF-INTA), Apdo 50727, \\ Madrid 28080 \\ email: crb@laeff.inta.es, esm@laeff.inta.es \\ ${ }^{4}$ Spanish Virtual Observatory (SVO), http://svo.laeff.inta.es
}

\begin{abstract}
The theory interest group in the International Virtual Observatory Alliance (IVOA) has the goal of ensuring that theoretical data and services are taken into account in the IVOA standards process. In this poster we present some of the efforts carried out by this group to include evolutionary synthesis models in the VO framework. In particular we present the VO tool PGos3, developed by the INAOE (Mexico) and the Spanish Virtual Observatory which includes most of public SSP models in the VO framework (e.g. VOSpec). We also describe the problems related with the inclusion of synthesis models in the VO framework and we try to encourage people to define the way in which synthesis models should be described. This issue has implications not only for the inclusion of synthesis models in the the VO framework but also for a proper usage of synthesis models.
\end{abstract}

Keywords. Galaxies: stellar content

\section{The VO and IVOA}

The Virtual Observatory (VO) is a system by which astronomers can produce more robust scientific results by allowing the use of multiple data centers (observational and theoretical ones), and by the development of analysis and visualization tools. The International Virtual Observatory Alliance (IVOA) $\dagger$ has the mission to facilitate the international coordination and collaboration necessary for the development and deployment of the VO.

One of the IVOA interest groups is the Theory interest group whose goal is to ensure that theoretical data and services are taken into account in the IVOA standard process.

\section{SSP models in the VO}

Currently, it is possible to access synthesis models in the VO framework. However, they are only implemented to access theoretical SEDs via Theoretical Spectral Access Protocol (TSAP) developed by ESA-VO and SVO, but TSAP does not cover all possible synthesis models results. Moreover, the access is only currently performed by spectral oriented tool (VOSpec $\ddagger$ in particular). Current services are illustrated in Fig. 1.

$\dagger$ http://www.ivoa.net

$\ddagger$ http://esavo.esa.int/vospec/ 


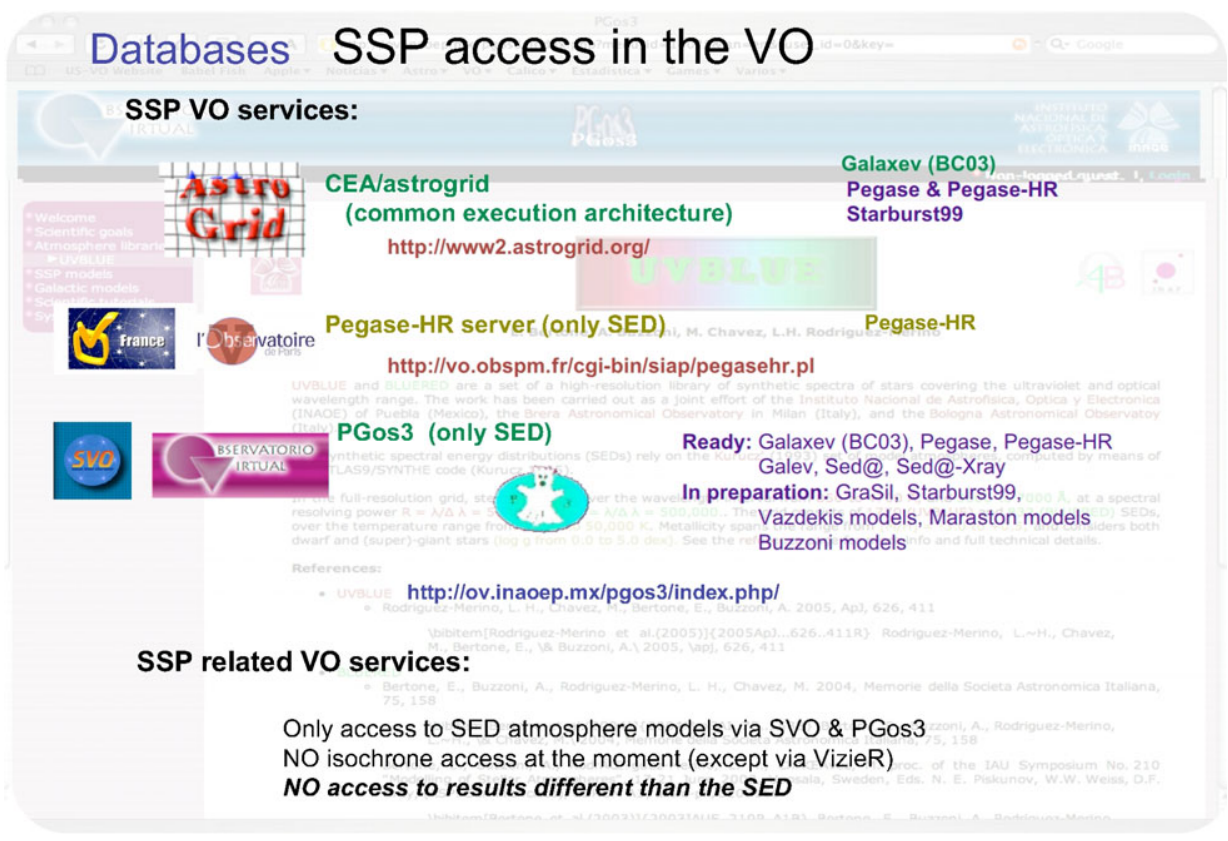

Figure 1. Schema of current SSP access services in the VO.

\section{The PGos3 database and Synthesis Models description}

PGos3 9 is a database aimed to provide SSP and related models in a VO framework for its later use in $\mathrm{VO}$ applications. It is included in a long term project for fitting and analysis of stellar populations in galaxies. PGos3 has been developed by the INAOE (Mexico) and the SVO, and it had been used for TSAP development.

However, to go further in the inclusion of synthesis models in the VO it is necessary to provide a Data Model of the possible results. That is, it is mandatory: (a) To define what means a synthesis code result: deterministic vs. probabilistic paradigm like Monte Carlo simulations or general probabilistic descriptions (Cerviño \& Luridiana (2006)). (b) To define how a synthesis model can be described univocally in terms of physical/computational parameters and not only code-authors names. And (c) to describe the limitations and parameter space coverage of different codes in an homogeneous way.

These requirements are not only VO requirements, but also scientific requirements needed (a) to understand why synthesis models computed by different codes with identical inputs differ, (b) to be able to establish physical justifications when a particular code, instead of a fashionable one, is chosen, and (c) to make a proper use of synthesis models.

\section{Acknowledgements}

This work was supported by the Spanish projects PNAYA2005-24102-E and PNAYA2004-02703. MC is supported by a Ramón y Cajal fellowship. VL is supported by a CSIC-I3P fellowship.

\section{Reference}

Cerviño, M. \& Luridiana, V. 2006, A\&A 451, 475

I http://ov.inaoep.mx/pgos3/ 